\title{
Clindamycin, Clotrimazole and Tinidazole in Mixed Vaginosis- A "Real World" Clinical Experience
}

\author{
Manish Maladkar*, Chitra Tekchandani, Urja Dave \\ Aristo Pharmaceuticals Pvt. Ltd., 23-A Shah Industrial Estate, Andheri, Mumbai, India
}

Email address:

scientific@aristopharma.org

To cite this article:

Manish Maladkar, Chitra Tekchandani, Urja Dave. Clindamycin, Clotrimazole and Tinidazole in Mixed Vaginosis- A "Real World" Clinical Experience. Journal of Gynecology and Obstetrics. Vol. 3, No. 3, 2015, pp. 49-54. doi: 10.11648/j.jgo.20150303.12

\begin{abstract}
Background: Vaginal infections are among the most frequent disorders affecting a woman and comprise over $95 \%$ of lower female genital tract infections. Seventy five percent of the women world-wide suffer from vaginosis resulting from bacterial, fungal and protozoal infections. Several studies have shown that mixed vaginal infections occur frequently and hence, in co-infection, each pathogen requires specific therapy for complete eradication of concurrent manifestations. Materials and Methods: A multicenter, prospective, open-label, non-comparative clinical study was carried out in 495 patients with mixed vaginosis of bacterial, fungal and protozoal origin. A fixed dose combination of Clindamycin $100 \mathrm{mg}$, Clotrimazole $100 \mathrm{mg}$ and Tinidazole $100 \mathrm{mg}$ was administered intravaginally for 7 consecutive nights. Results: Treatment led to significant reduction in various symptoms of vaginal infection within 3 days of treatment initiation. On day 7 of treatment, $98.6 \%$ patients had normal and odourless vaginal discharge, the mean vaginal burning and irritation score declined by $99.2 \%$ and $99.3 \%$ respectively. Also, there was remission of vulvar, cervical and vaginal erythema in more than $98 \%$ of patients. Physicians and patients rated the treatment efficacy as good to very good improvement and tolerability to the treatment was regarded as good to excellent. No serious adverse events were reported. Conclusion: The present study demonstrates that fixed dose combination of Clindamycin, Clotrimazole and Tinidazole is effective and well tolerated in the treatment of mixed vaginosis due to bacterial, fungal and protozoal infection.
\end{abstract}

Keywords: Clindamycin, Clotrimazole, Tinidazole, Vaginosis, Candidiasis, Trichomoniasis

\section{Introduction}

Vaginosis is among the most common conditions for which women seek medical consultation, with vaginal discharge accounting for frequent clinic visits ${ }^{1}$. Throughout their lifetime, $75 \%$ of women are estimated to suffer from at least one episode of vaginosis ${ }^{2}$. Symptoms of vaginosis can cause substantial distress to the patient, resulting in time lost from work and lowering of self-esteem ${ }^{3}$. Vaginal infections not only cause suffering, but their consequences are far more devastating. These infections present a huge burden of disease and adversely impact the reproductive health of women ${ }^{4}$.

Common infectious forms of vaginosis include bacterial vaginosis $(\mathrm{BV})$, vulvovaginal candidiasis and trichomoniasis ${ }^{5}$. These should be treated effectively to reduce the risk of acquisition of other sexually transmitted infections since vaginosis is linked with decreased levels of protective Lactobacilli and increased local inflammation ${ }^{6}$.
Bacterial vaginosis is associated with an increased risk of upper genital tract infections e.g. pelvic inflammatory disease that comprises of cervicitis, endometritis, salpingitis, oophoritis, pelvic abscess, septic thrombophlebitis, etc. Vulvovaginal candidiasis, the other commonest cause of infective vaginal discharge, has adverse effects on fertility. Also, trichomoniasis augments the risk of pelvic inflammatory disease leading to infertility ${ }^{7}$.

Several studies have shown that mixed vaginal infections occur frequently ${ }^{8,9,10,11}$. Presence of mixed vaginal infections and bacteria from the rectum that is likely to colonize the vagina are responsible for increased risk of re-infections. High incidence of recurrence and repeated use of antimicrobial agents whose effectiveness is diminishing due to upsurge of microbial resistance has a significant negative impact on the patient's quality of life ${ }^{12,13}$.

Management of vulvovaginitis is very challenging since 
the diagnosis of vaginosis by common methods is not precise and use of specific methods is time consuming and often expensive ${ }^{14}$. There is high percentage of women in whom it is difficult to establish the etiological agent and/or in whom the infection is diagnosed as mixed. Thus, there is a dire need for syndromic management of vaginosis that covers the principal causes of vaginosis and allows management of patients at the time of first contact.

Oral and vaginal antimicrobial therapy are the available options for the treatment of bacterial vaginosis, however, the use of oral therapy is constrained by the potential for systemic adverse effects. In clinical practice, local antibiotic therapy is preferred for the routine treatment of BV. The recommended antibacterial agents for BV include Metronidazole and Clindamycin. Literature offers a number of reports revealing higher cure rates and lower resistance with Clindamycin than Metronidazole ${ }^{15,16}$.

Vulvovaginal candidiasis is effectively treated with topical azoles including Clotrimazole that results in relief of symptoms and negative cultures in $80-90 \%$ of patients who complete the therapy ${ }^{17}$. Treatment of patients with Metronidazole-refractory vaginal trichomoniasis constitutes a major therapeutic challenge and limited treatment options emphasize the use of potent trichomonicidal agent like Tinidazole.

The current study investigated the intravaginal effectiveness and safety of the fixed dose combination of Clindamycin, Clotrimazole and Tinidazole in the treatment of mixed vaginosis.

\section{Materials and Methods}

\subsection{Design of Investigation}

A prospective, open-label, non-comparative and multicentric study included 495 women with clinical diagnosis of mixed vaginosis. The study was conducted at 5 different centers in India by qualified investigators. Demographic data, medical history, previous treatment and physical examination data were collected from all enrolled patients at baseline (visit I). Treatment effectiveness was assessed at visit II (day 3) and visit III (day 7). Efficacy evaluation was carried out based on improvement in parameters like consistency and odour of vaginal discharge, vaginal burning, vaginal irritation, vaginal redness, vulvar erythema and cervical erythema. Safety assessment was carried out depending upon the occurrence of adverse event, if any and its severity. At the end of the treatment, global assessment of efficacy and tolerability was confirmed by the physicians and patients.

\subsection{Study Medication}

Soft gelatin capsule containing fixed dose combination of Clindamycin $100 \mathrm{mg}$, Clotrimazole $100 \mathrm{mg}$ and Tinidazole $100 \mathrm{mg}$ (Clingen Forte ${ }^{\circledR}$ ) was administered intravaginally for 7 consecutive nights.

\subsection{Patient's Selection}

Inclusion Criteria: Females 18 years of age or older with clinical diagnosis of mixed vaginosis of bacterial, fungal and protozoal origin and not responding to other therapies.

Exclusion Criteria: History of hypersensitivity to Clindamycin, Clotrimazole or Tinidazole; pregnant or lactating women, menstruating women or expected to be menstruating within days of starting study medication. Also, women receiving any antibiotic treatment 14 days prior to enrolment were excluded from the study.

The enrolled subjects agreed not to douche or use any intravaginal products during the study period.

\subsection{Clinical Assessment Parameters}

The efficacy of the combination was evaluated at day 3 and day 7 after treatment initiation. Vaginal discharge of patients was assessed for: (i) consistency- white, thin, flocculent (normal); white curdy discharge; thin greyishwhite homogeneous discharge coating the vaginal walls; yellow-green frothy discharge and (ii) odour- fishy, offensive or odourless. Occurrence of vaginal burning and irritation was recorded and graded as mild, moderate or severe. Presence or absence of other symptoms like vaginal redness, vaginal erythema, vulvar erythema and cervical erythema was noted. Evaluation of safety was depending on adverse event, if any, reported by the patient or observed by the investigator. The intensity of adverse events was graded as mild, moderate or severe and the onset of adverse effects was characterized as sudden, gradual or unknown.

The global assessment of efficacy of treatment was conducted by the physicians and patients at the end of the study based on the scale: 1 = very good improvement, $2=$ good improvement, 3 = moderate improvement, $4=$ negligible improvement; and tolerability was assessed depending on the scale: $1=$ excellent, $2=\operatorname{good}, 3=$ fair, $4=$ poor.

\subsection{Statistics}

The data was pooled and the results were analyzed using parametric and non-parametric tests. All tests were two tailed and $p<0.05$ was considered significant.

\section{Results}

\subsection{Demographic Profile}

A total of 495 cases were evaluated in the study. The overall demographic breakup of the patients is depicted in table 1 . The range of patients' age and weight was 20-45 years and 43-72 kg respectively. Also, physical examination parameters such as temperature, pulse rate, respiratory rate and blood pressure were within normal limits at baseline (table 2). 
Table 1. Demographic Data

\begin{tabular}{ll}
\hline Parameters & \\
\hline No. of cases & 495 \\
Age (yrs) & \\
Mean & 32.18 \\
SD & 5.58 \\
Range & $20-45$ \\
Weight $(k g)$ & \\
Mean & 62.07 \\
SD & 5.18 \\
Range & $43-72$ \\
\hline
\end{tabular}

Table 2. Profile of Physical Examination

\begin{tabular}{ll}
\hline Physical Examination & (Mean \pm SD)(N=495) \\
\hline Temperature $\left({ }^{\circ} \mathrm{F}\right)$ & $97.87 \pm 0.80$ \\
Pulse rate $(/ \mathrm{min})$ & $75.45 \pm 1.74$ \\
Respiratory rate $(/ \mathrm{min})$ & $17.13 \pm 2.70$ \\
SBP $(\mathrm{mmHg})$ & $119.40 \pm 5.07$ \\
DBP $(\mathrm{mmHg})$ & $90.39 \pm 5.25$ \\
\hline
\end{tabular}

\subsection{Efficacy Evaluation}

\section{Effect on Vaginal Discharge Consistency:}

At baseline, all patients had abnormal vaginal discharge. Yellow-green frothy discharge was noted in 347 patients; 93 patients presented with thin, greyish-white homogeneous discharge coating the vaginal walls and 55 patients had white curdy discharge. On visit II (day 3 ) after treatment initiation, $82.4 \%$ women had normal vaginal discharge and on visit III (day 7), the patients with normal vaginal discharge increased to $98.6 \%$. Thus, statistically significant number of patients benefited with treatment, $\mathrm{p}<0.05$ (figure 1).

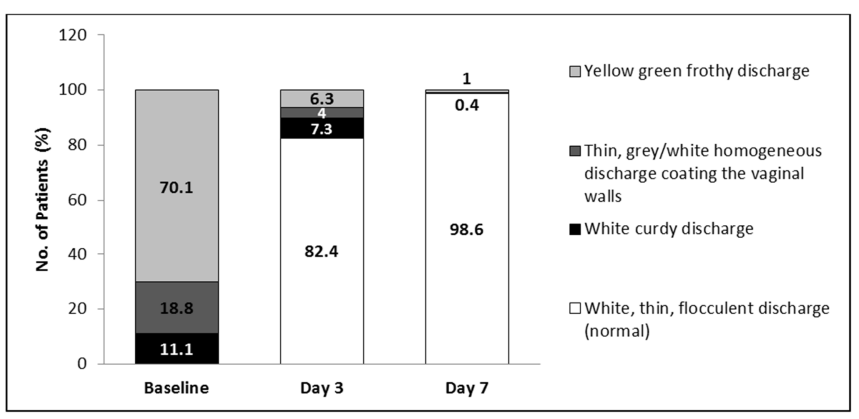

Figure 1. Changes in Number of Cases with Vaginal Discharge Consistency

\section{Effect on Odour of Vaginal Discharge:}

All the patients enrolled for the study had vaginal discharge with fishy or offensive odour at baseline. Post treatment, statistically significant number of patients reported odourless vaginal discharge. On visit II, $64.8 \%$ patients had odourless vaginal discharge which further increased to $98.6 \%$ on day 7 of treatment (figure 2).

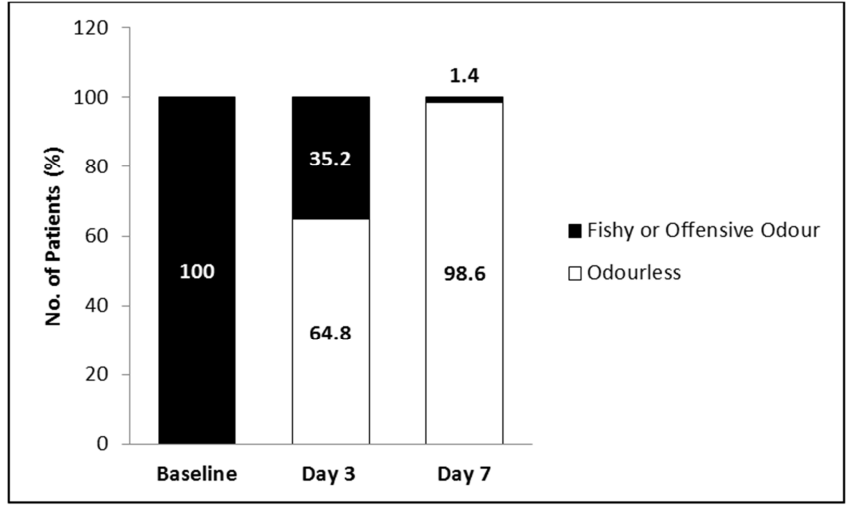

Figure 2. Changes in Number of Cases with Vaginal Discharge Odour

\section{Effect on Vaginal Burning and Vaginal Irritation:}

The mean score of vaginal burning was 1.35 at baseline. At visit II and III, the mean score of vaginal burning showed a significant fall of $57 \%$ and $99.3 \%$ from baseline respectively, $p<0.05$.

According to the analysis, the mean score of vaginal irritation was 1.35 at baseline. A significant decline of $63 \%$ and $99.3 \%$ was noticed at day 3 and 7 post treatment, $p$ $<0.05$ (figure 3).

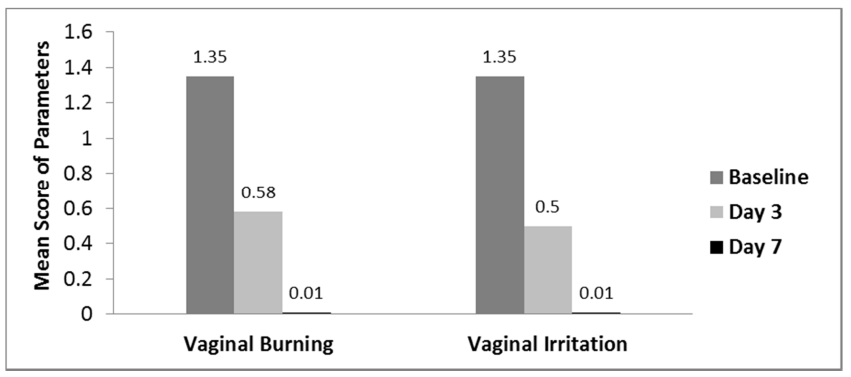

Figure 3. Changes in Mean Score of Vaginal Burning and Vaginal Irritation

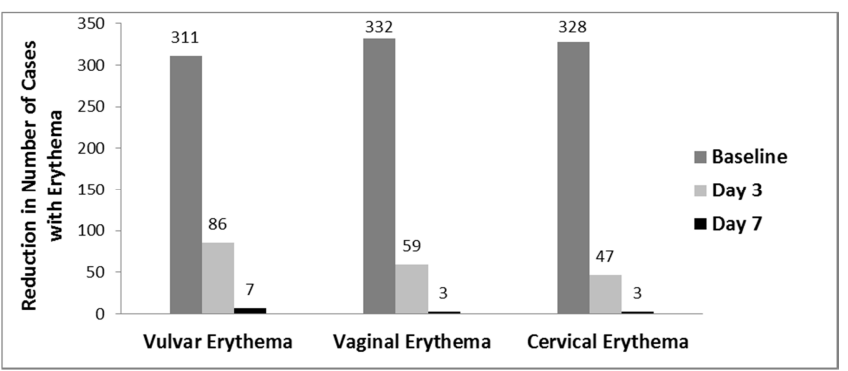

Figure 4. Changes in Number of Cases with Vulvar, Vaginal and Cervical Erythema

\section{Effect on Erythema}

- Vulvar Erythema

At baseline, $62.8 \%$ patients reported vulvar erythema. On visit II, only $17.4 \%$ presented with vulvar erythema and $98.6 \%$ of patients reported absence of vulvar erythema on day 7 . Thus, a statistically significant increase was observed in number of patients reporting resolution of vulvar erythema post treatment, $p<0.05$ (figure 4). 
- Vaginal Erythema:

Number of patients presenting with vaginal erythema at baseline was 332. After treatment, $88.1 \%$ and $99.4 \%$ of patients reported absence of vaginal erythema on visit II and III, respectively. Thus, patients exhibiting vaginal erythema reduced considerably at the end of treatment, $p<0.05$ (figure 4).

\section{- Cervical Erythema:}

Patients with cervical erythema at baseline were found to be 328 . At visit II, substantial decline of $90.5 \%$ was noticed in the number of patients with cervical erythema and at visit III, $99.4 \%$ of patients were free of cervical erythema, $p<0.05$ (figure 4).

\section{Global Assessment of Efficacy of Treatment:}

As per physicians' evaluation, $82 \%$ of the cases showed very good improvement and remaining $18 \%$ showed good improvement after the treatment (figure 5a). According to patients' evaluation, $82.2 \%$ of the patients had very good and $17.8 \%$ had good improvement after the treatment (figure $5 \mathrm{~b}$ ).

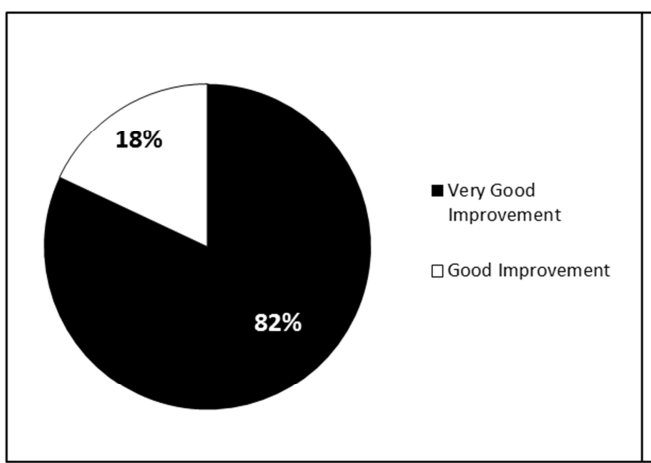

(a)

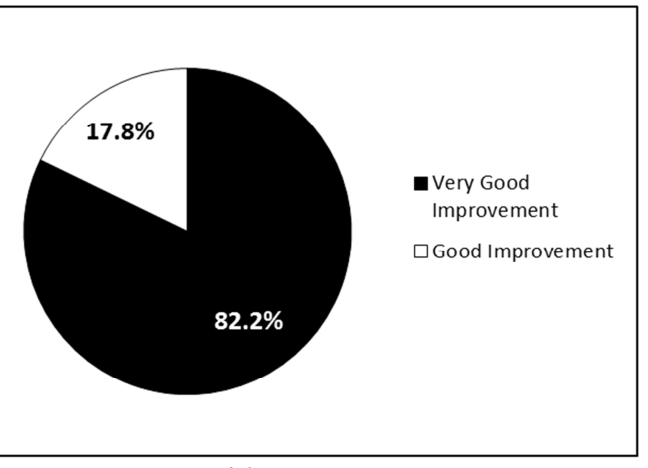

(b)

Figure 5. Overall global assessment of efficacy of treatment by physicians (a) and patients (b)

6. Global Assessment of Tolerability of Treatment:

As per physicians' evaluation, $95.4 \%$ of the cases showed excellent and $4.6 \%$ had good tolerability of the treatment (figure 6a) and according to patients' evaluation, 94.9\% of them had excellent while $5.1 \%$ had good tolerance to the treatment (figure 6b).

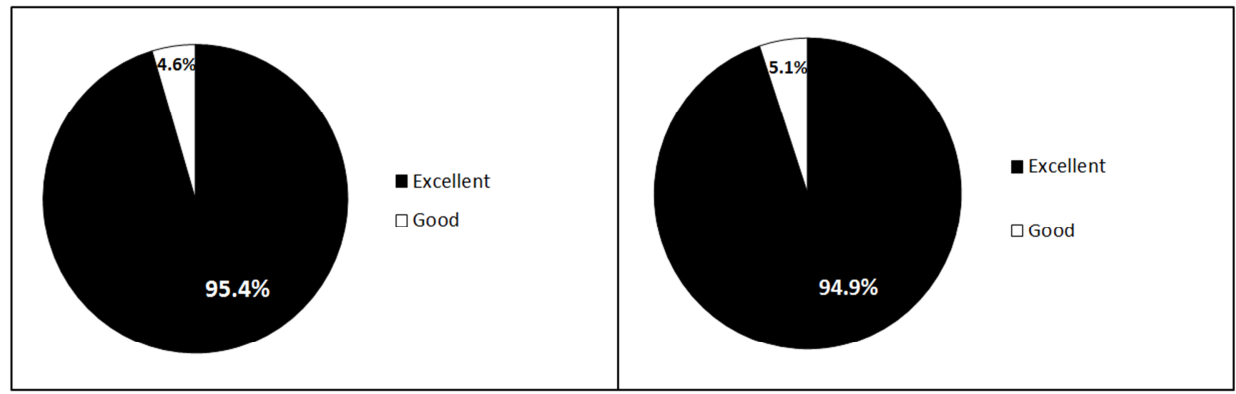

(a)

(b)

Figure 6. Overall global assessment of tolerability of treatment by physicians (a) and patients (b)

Table 3. Profile of Adverse Events

\begin{tabular}{ll}
\hline Adverse events & Percentage of patients \\
\hline Abdominal cramps & 0.2 \\
Burning & 1.0 \\
Diarrhea & 0.2 \\
Frequent urination & 0.6 \\
Headache & 0.2 \\
Irritation & 0.6 \\
Itching & 1.0 \\
Rash & 0.2 \\
Vaginal Pain & 0.8 \\
Total number of events & 4.8 \\
Total number of cases & 4.6 \\
\hline
\end{tabular}

7. Safety Assessment:

In the present study $4.6 \%$ of the total cases had adverse events (AEs) (table 3). The reported AEs were mild to moderate in all the cases and onset of adverse events was unknown. Most of the AEs were tolerable and disappeared during treatment. There was no known relationship of adverse events to the study medication.

\section{Discussion}

Vaginal infection is a common polymicrobial syndrome reported among women of various age groups. The normal vaginal microflora consists of Lactobacilli, while the disturbed vaginal microflora is characterized by the overgrowth of bacteria like Gardnerella vaginalis, Mobiluncus spp., Mycoplasma hominis, Prevotella spp. and protozoa viz. Trichomonas vaginalis. Also, it is documented 
that vaginal infections due to Candida spp. has been increasing over the decades ${ }^{18}$.

In view of the mixed etiologies of vaginal infection and the inability to carry out comprehensive assessment of patients, syndromic management would be a valuable form of therapy that is aimed at treating all the likely causes of vaginosis and also would reduce the recurrence rate ${ }^{19}$.

Systemic or local antibiotic therapy remains the standard regimen for the treatment of infective vaginosis. The treatment regimens recommended by the Centre for Disease Control and Prevention (CDC) for bacterial vaginosis are either Clindamycin or Metronidazole ${ }^{20}$. Therapeutic options for practitioners in the treatment of BV have increased from oral agents to better tolerated and more preferred topical treatments.

Clotrimazole is a potent antifungal agent against Candida species as well as effective against Trichomonas vaginalis ${ }^{21}$. It has been widely prescribed for topical or intravaginal use in vulvovaginal candidiasis. Further, addition of the Clotrimazole to a nitroimidazole derivative like Tinidazole, would improve the antitrichomonal action of the latter.

The nitroimidazoles comprise the only class of drugs useful for the treatment of trichomoniasis. Of these agents, Metronidazole and Tinidazole are employed in the treatment of trichomonal vaginosis. Metronidazole-resistant trichomoniasis had been observed within two years of Metronidazole introduction, when used for this indication. Treating patients refractory to Metronidazole is extremely challenging for physicians and is associated with intense patient suffering. Unlike Metronidazole, the recommended Tinidazole regimen has resulted in cure rates of upto $100 \%{ }^{17}$. In a study involving Metronidazole-resistant vaginal trichomoniasis, vaginal Tinidazole has shown to be successful in $92 \%$ of the patients ${ }^{22}$.

The present study was undertaken to evaluate efficacy and safety of fixed dose combination of Clindamycin, Clotrimazole and Tinidazole in the management of mixed vaginosis. The evaluation parameters included change in vaginal discharge consistency and odour; change in the number of cases reporting vaginal, vulvar and cervical erythema and improvement in the scores of vaginal burning and irritation.

Favourable clinical responses were demonstrated in majority of the treated patients. Gynecological assessment showed statistically significant $(p<0.05)$ improvement with combination therapy in troublesome symptoms of vaginal infection i.e. burning and irritation of pelvic region. The severity of vaginal burning had reduced by $99.3 \%$ within 7 days of treatment. Majority of patients had near normal scores of vaginal irritation at the follow-up visits.

Treatment with combination therapy provided prompt onset of relief with regards to disappearance of clinical indicators of vaginosis. Beneficial effects were exhibited within 3 days of study commencement and complete eradication of infection was noticed in preponderance of patients by day 7 of the study.

Statistically significant number of patients exhibited improvement in vaginal manifestations. The conversion of pathological vaginal discharge to normal consistency was observed in $98.6 \%$ of women. The transformation of malodorous vaginal discharge to odourless was reported in $98.6 \%$ patients by the end of the treatment. More than $99 \%$ of patients had improvement in vaginal burning and irritation at study completion. Also vulvar, vaginal and cervical erythema disappeared in almost every patient enrolled for the study.

Overall treatment efficacy was rated as very good not only by the physicians, but by the patients too. The beneficial effects of combination therapy were due to comprehensive spectrum of antibacterial, antifungal and antiprotozoal action.

The combination was well tolerated with no major adverse effects being experienced by the patients. The intensity of these effects was described as mild to moderate and resolved during the course of treatment.

\section{Conclusion}

The clinical trial confirms the intravaginal use of fixed dose combination of Clindamycin, Clotrimazole and Tinidazole as the prospective therapy to cover the most common etiologies of vaginosis. The combination ensures a wider range of antimicrobial action with rapid and noteworthy symptomatic relief in patients with mixed vaginosis of bacterial, fungal and protozoal origin. Also, the therapy is found to be tolerable with no serious safety concerns. Thus, the fixed dose combination represents a significant advance in the treatment of vaginal infection of mixed origin.

\section{References}

[1] Marion KO, Timothy LC. Management of Vaginitis. American Family Physician. 2004; 70: 2125-32.

[2] Fabio P, Aroldo C, Geraldo D, Lara L, Luis B, Alvaro P. Efficacy and tolerance of metronidazole and miconazole nitrate in treatment of vaginitis. International Journal of Gynecology and Obstetrics. 2008; 102: 287-292.

[3] Linda OE. Acute Vulvovaginitis. New England Journal of Medicine. 2006; 355: 1244-52.

[4] Divya AP, Nancy MB, Kathryn MC. Reproductive Tract Infections. Centers for Disease Control and Prevention. 2003; module 3: $1-83$.

[5] Tahniat SS, Paula KB. Vaginitis in adolescents. Adolescent medicine clinics. 2004; 15: 235-251.

[6] Jyoti T, Alka K, Nutan A, Sreenivas V. Aetiology \& risk factors of recurrent vaginitis \& its association with various contraceptive methods. Indian Journal of Medical Research. 2010; 131: 83-87.

[7] Katz, Lentz, Lobo, Gershenson. Comprehensive Gynecology, 5th ed., 2007. 
[8] Prashini M, Cathy C, Willem AS. Interrelationships among Human Immunodeficiency Virus Type 1 Infection, Bacterial Vaginosis, Trichomoniasis, and the Presence of Yeasts. The Journal of Infectious Diseases. 2002; 185: 69-73.

[9] Líbalová Z, Cepický P, Malina J, Stanslický K, Kuzelová M, Medalová Z, et al. Vulvovaginitis. Occurrence and importance of mixed and unclassifiable Pictures. Ceská Gynekologie. 2007;72:32-7.

[10] Karaca M , Bayram A, Kocoglu ME, Gocmen A, Eksi F. Comparison of clinical diagnosis and microbiological test results in vaginal infections. Clinical and Experimental Obstetrics and Gynecology. 2005; 32: 172-4.

[11] Cepický P, Malina J, Líbalová Z, Kuzelová M. Mixed" and "miscellaneous" vulvovaginitis: diagnostics and therapy of vaginal administration of nystatin and nifuratel. Ceská Gynekologie. 2005;70:232-7.

[12] Reid G, Bruce A W. Urogenital infections in women: can probiotics help? Postgraduate Medical Journal. 2003;79:42832.

[13] Sarah R, Natasha A, Gillian S, Andrew W. Practical management of recurrent vulvovaginal candidiasis. Trends in Urology Gynaecology \& Sexual Health. 2009; 14: 18-32.

[14] E O“zyurt, MB Toykuliyeva, IL Danilyans, O Morton, G Baktir. Efficacy of 7-day treatment with metronidazole + miconazole (Neo-Penotran) a triple-active pessary for the treatment of single and mixed vaginal infections. International Journal of Gynecology \& Obstetrics. 2001; 74: 35-43.

[15] Nagaraja P. Antibiotic resistance of Gardnerella vaginalis in recurrent bacterial vaginosis. Indian Journal of Medical Microbiology. 2008; 26:155-7.
[16] Tomusiak A, Strus M, Heczko PB. Antibiotic resistance of Gardnerella vaginalis isolated from cases of bacterial vaginosis. Ginekologia Polska. 2011;82:900-4.

[17] Kimberly A. Workowski MD, Stuart M B. Sexually Transmitted Diseases Treatment Guidelines. Centers for Disease Control and Prevention. 2006; 55: 1-94.

[18] Sheila JA, Rodrigo VL, Zahir T, Elias S, Maria LG, Maria AH et al. Frequency of Trichomonas vaginalis, Candida sp and Gardnerella vaginalis in cervical-vaginal smears in four different decades. Sao Paulo Medical Journal.2001; 119: 2005 .

[19] Jacques P, François S, Nzambi K, Thomas AP, Soumaila $\mathrm{D}$, Sylvie $\mathrm{D}$ et al. The syndromic management of vaginal discharge using single-dose treatments: a randomized controlled trial in West Africa. Bull World Health Organ. 2006; 84: 729-738.

[20] Kimberly A. Workowski MD, Stuart M B. Sexually Transmitted Diseases Treatment Guidelines. Centers for Disease Control and Prevention. 2010; 59: 1-110.

[21] Anjali Aggarwal, R. Michael Shier. Recalcitrant Trichomonas Vaginalis Infections Successfully Treated With Vaginal Acidification. Journal of Obstetrics and Gynaecology Canada . 2008; 30:55-58.

[22] J. D. Sobel, P. Nyirjesy, William Brown. Tinidazole Therapy for Metronidazole-Resistant Vaginal Trichomoniasis. Clinical Infectious Diseases. 2001; 33:1341-6. 